\title{
PARIS AGREEMENT
}

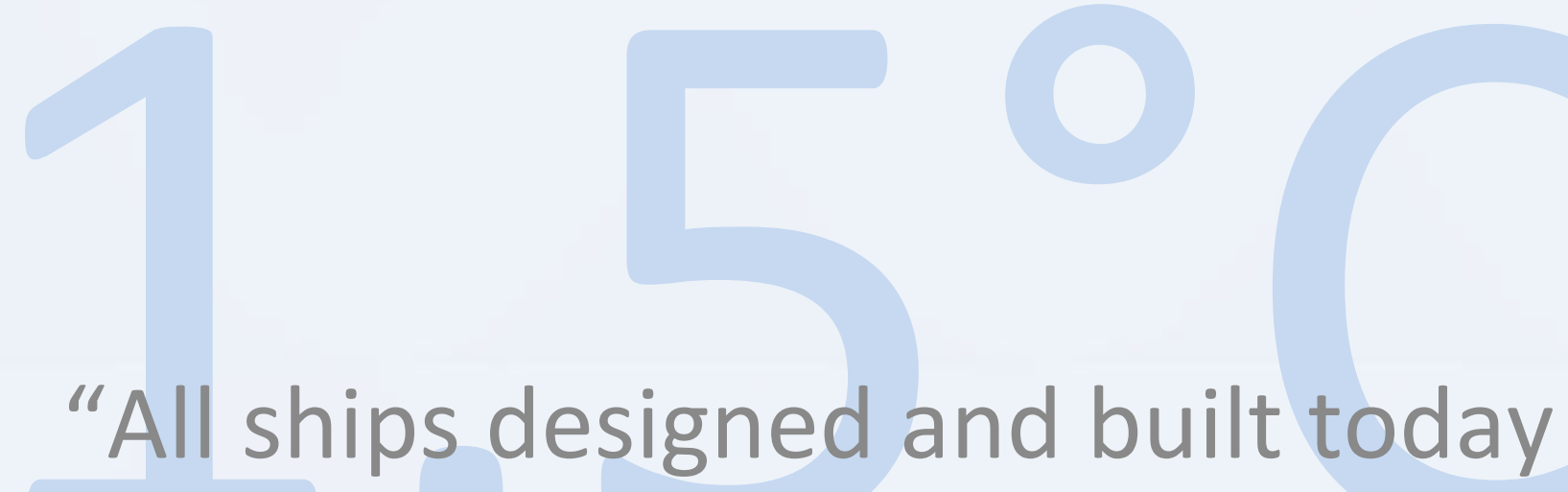

must operate in a net zero emissions world at the end of their service life" 


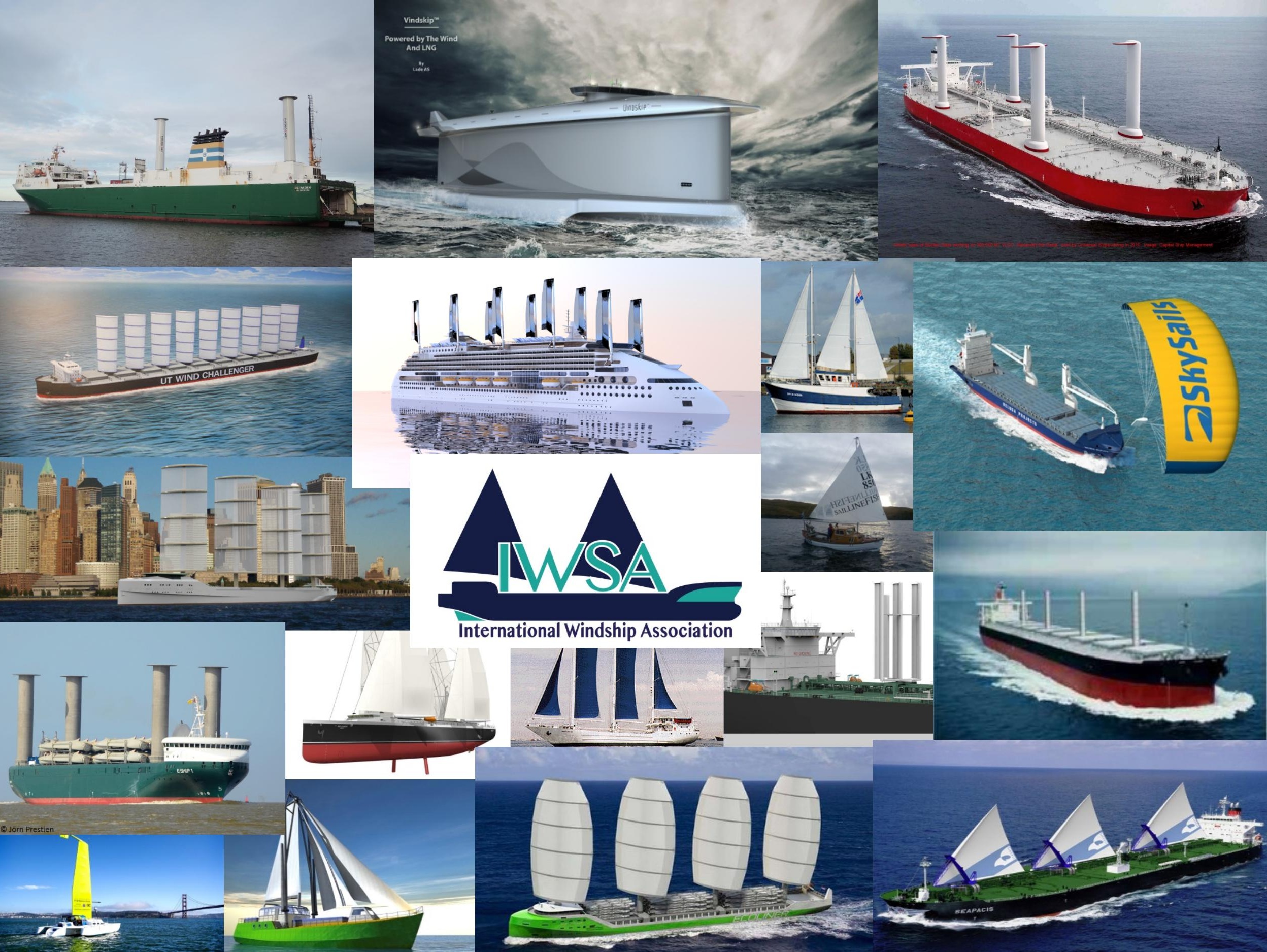




\section{Challenges Facing Shisping}

Regulation \& Compliance Costs (SOx, EEDI, SEEMP etc.)

Future Regulatory Framework? (GHG, NOx, Noise, PM, BC)

Regional Legislation (EU, US)

Volatility in Fuel Prices \& Energy Security

Split Incentives for Efficiency Investments

Next Generation Fuels?

Technology Selection

Over Capacity

Future Trade Patterns \& Prediction

Crewing \& Training

And so on........ 


\section{Uncertainty}

Transition

Volatility 


\section{Wind Propulsion Systems can.....}

- $\quad$...be retrofitted to existing vessels or incorporated into new build design.

- ...reduce dependency on fossil fuels and deliver increased energy security.

- ...deliver $10-30 \%$ fuel savings as retrofit and around $50 \%$ for new builds.

- ...help make vessels compliant with existing regulation and prepare for future scenarios.

- ...increasingly be viewed as a credible, viable and costeffective alternative.

"In 2030, the market potential could amount to 3,700-10,700 installed systems on bulkers \& tankers, associated with approx. 3.5-7.5 Mt CO2 savings \& 6,500-8,000 direct + 8,500-10,000 indirect jobs." EU Report Nov 2016 


\section{Wind Propulsion Technology Options?}

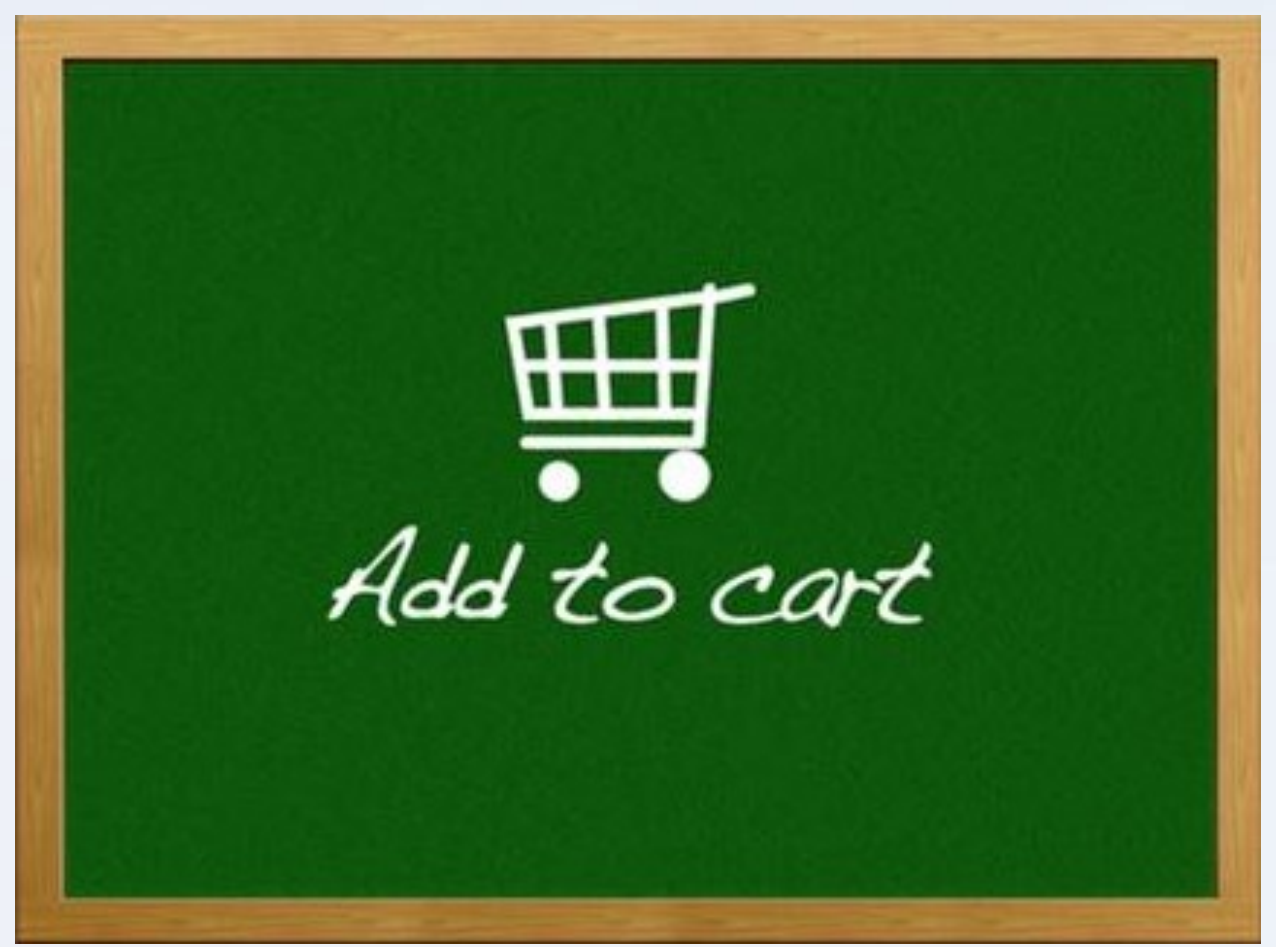




\section{Technology Toolbox}

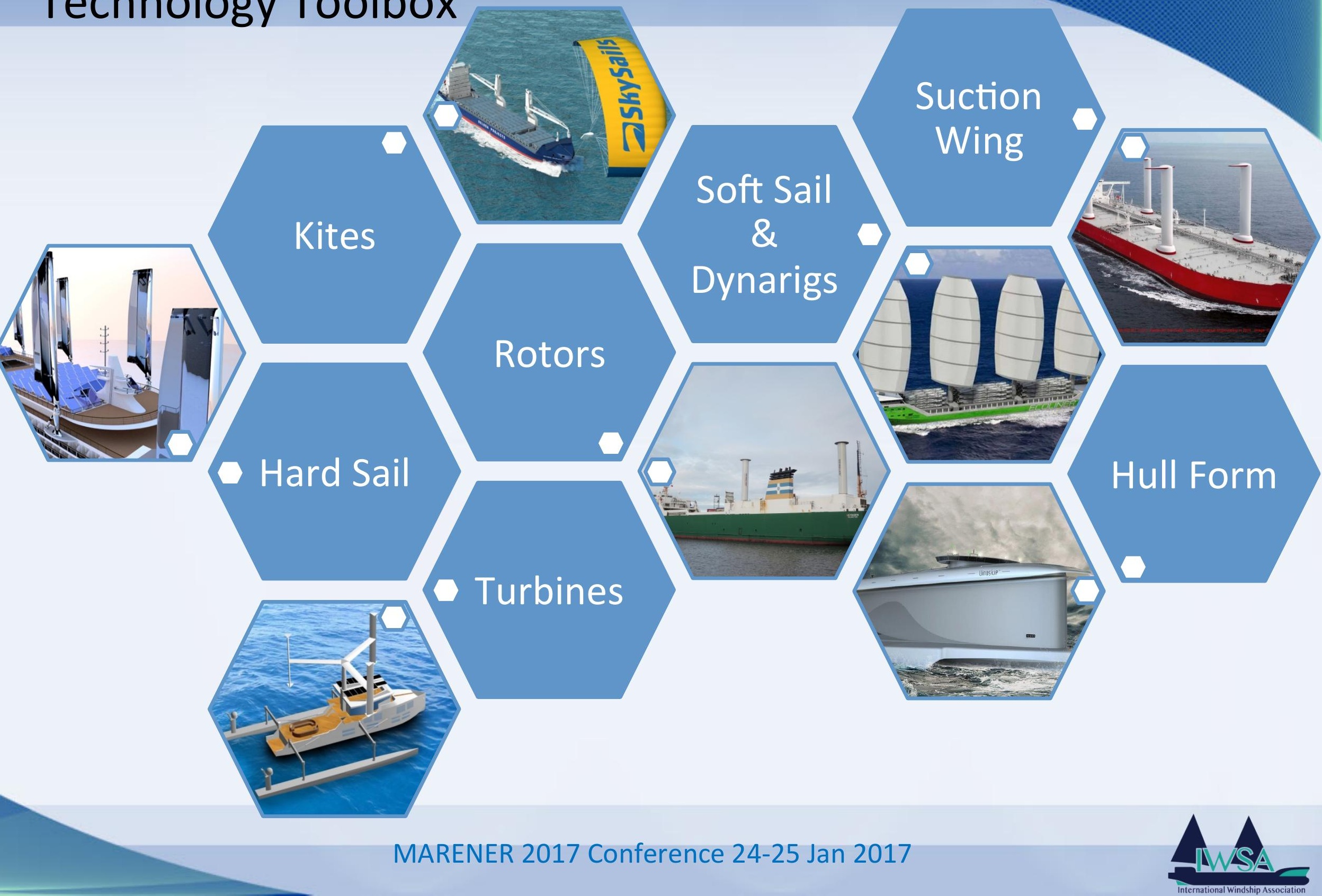




\section{Stage of Development?}

\begin{tabular}{|c|c|c|c|c|c|}
\hline Technology & Ship Size & 2008 & 2014 & 2016 & 2018 \\
\hline \multirow{2}{*}{ Rotors } & $<10,000 \mathrm{t}$ & & & & \\
\hline & $>10,000 t$ & & & & \\
\hline \multirow{2}{*}{ Hard Sail } & $<10,000 t$ & & & & \\
\hline & $>10,000 \mathrm{t}$ & & & & \\
\hline \multirow{2}{*}{ Soft Sail } & $<10,000 \mathrm{t}$ & & & & \\
\hline & $>10,000 t$ & & & & \\
\hline \multirow[t]{2}{*}{ Kite } & $<10,000 \mathrm{t}$ & & & & \\
\hline & $>10,000 t$ & & & & \\
\hline \multirow[t]{2}{*}{ Suction Wing } & $<10,000 t$ & & & & \\
\hline & $>10,000 \mathrm{t}$ & & & & \\
\hline \multicolumn{6}{|l|}{ Turbine } \\
\hline \multicolumn{6}{|l|}{ Hull Form } \\
\hline \multicolumn{2}{|c|}{ Concept } & $R \& D$ & & Test \& Trial & Commercial \\
\hline
\end{tabular}




\section{IWSA Membership \& Windship Network}

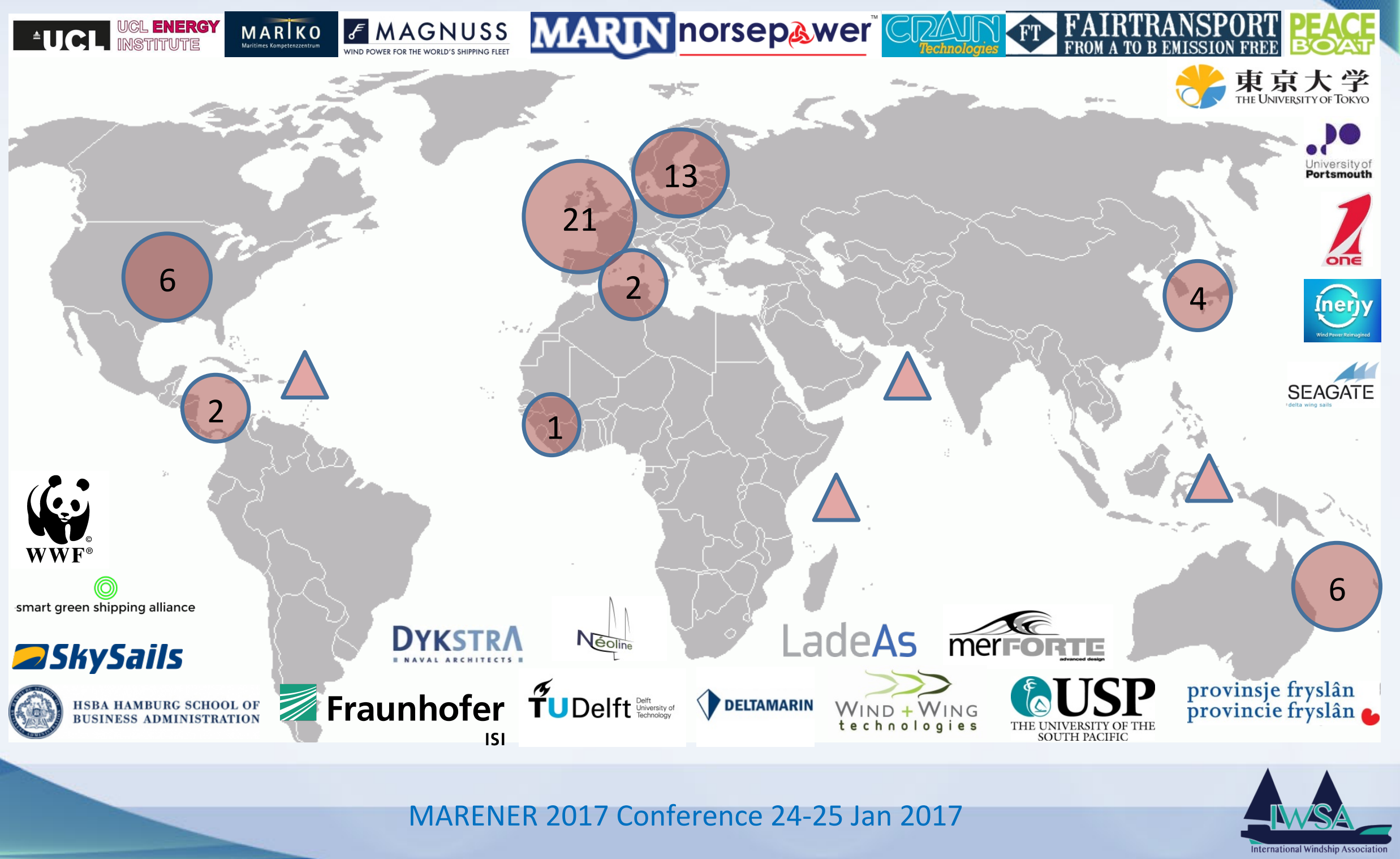




\section{IWSA Work Stream Development}

"Facilitate and promote wind propulsion for commercial shipping worldwide and bring together all parties in the development of a Windship sector."

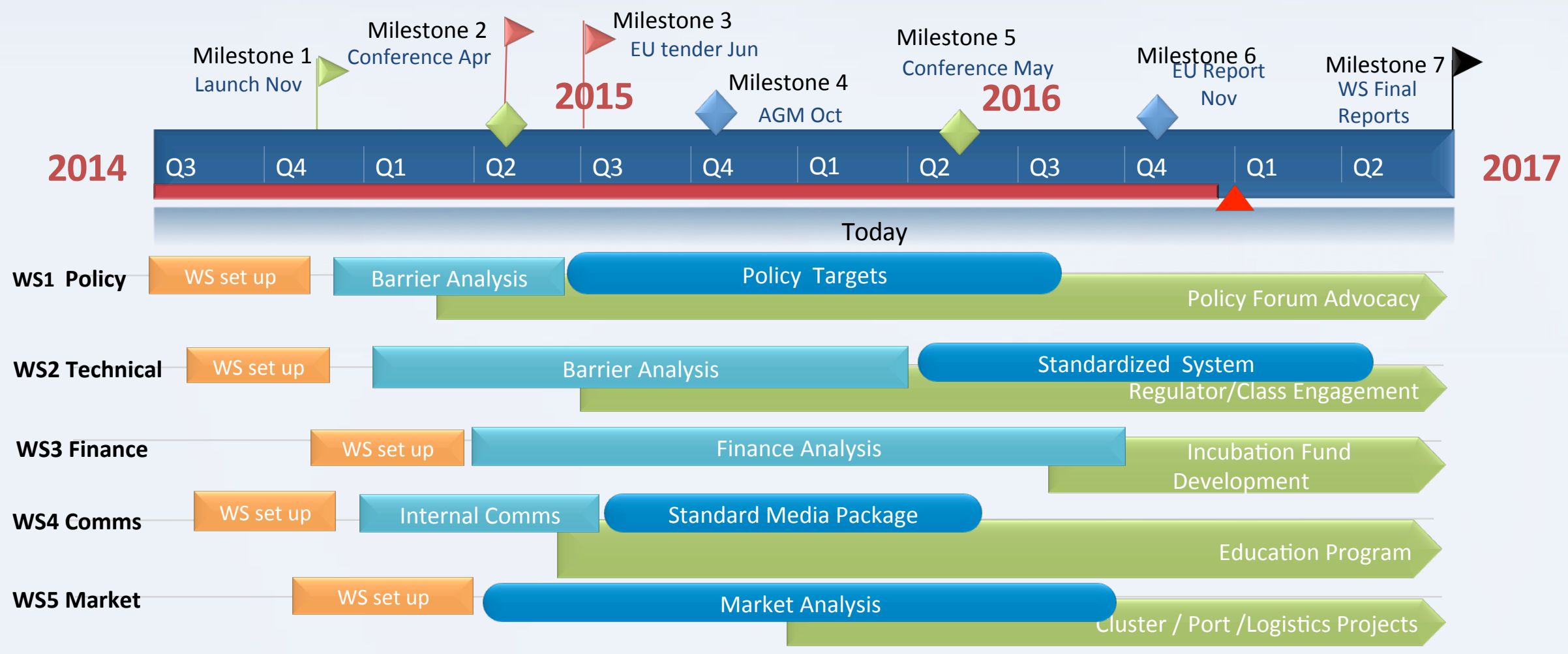




\section{Barriers \& Challenges}

Industry structure - the split incentive, charter party clauses

Solution - new mechanisms e.g. SSI2040 Save as you Sail, Clean Shipping Index, CWR

Perception - a psychological barrier on a potential solution that is so visible.

Solution - increasing number of demonstration vessels, communication.

The promises - the need to 'prove' savings

Solution - more demo vessels, independent verification, pooling of information (IWSA)

Capital intensity for working demonstrators - cost for technology to reach market Solution - increasing coop/pooling resources (IWSA), finance facility \& build tech cluster.

Lack of technology transfer - from the offshore and yacht sectors.

Solution - increasing transfer is underway, incubation/innovation support (IWSA)

Operational \& technical - route specific savings, compliance, cargo handling etc. Solution - working with class, NA/engineers. Weather routing, automated systems, operations management etc.

Lloyds Register: Wind-powered shipping 2015 


\section{Case Study: Rotor Development}

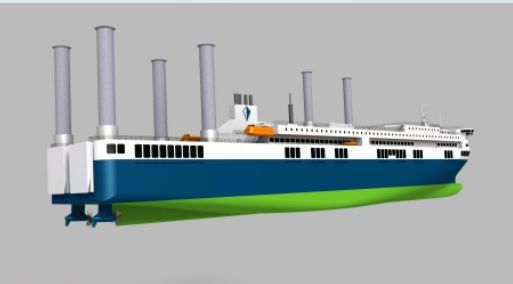

Deltamarin Award winning 'Delta Challenger' design

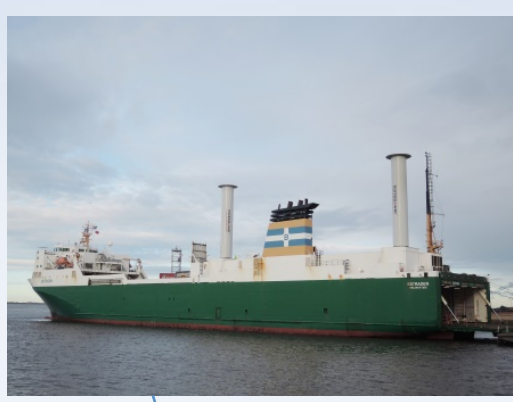

Norsepower fits twin rotors on M/V Estraden $\checkmark$
EU Fünding Large Rotor R\&D

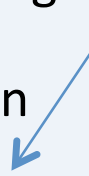

\section{Itaccoscan 1}

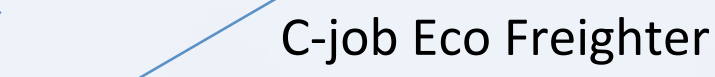

Design Contract

\section{9}

$\uparrow$

Enercon's E-ship 1 operational

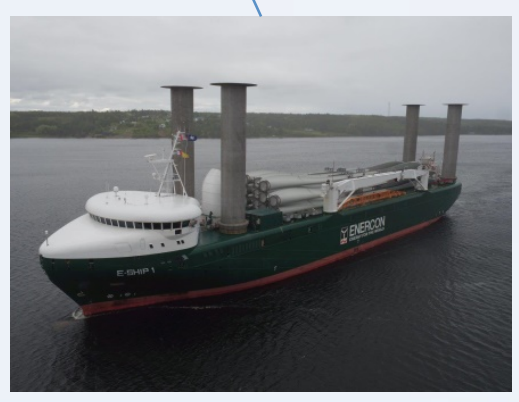

\section{4}

$\uparrow$ Wind Hybrid Coaster Design

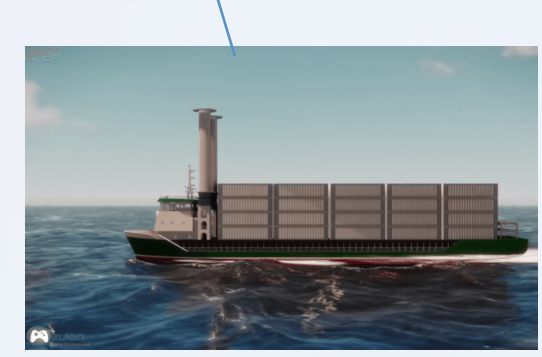

2016
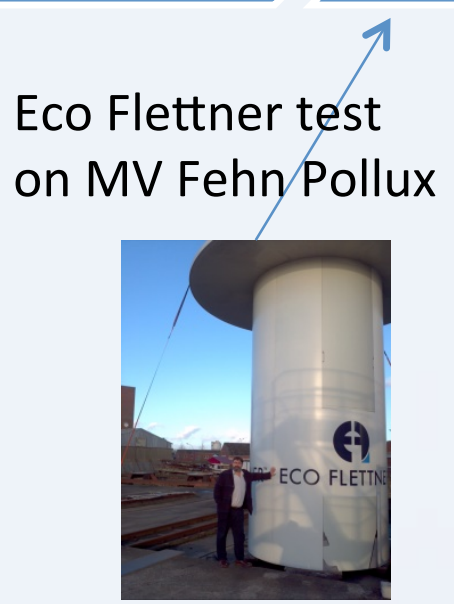

\section{7}

Magnuss

Commercial Test Rig

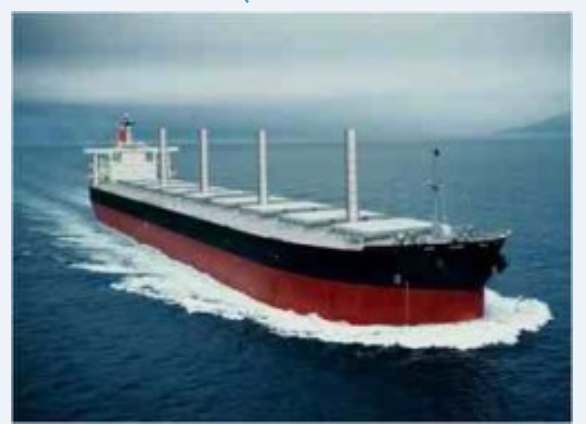




\section{Case Study: Wind Challenger}

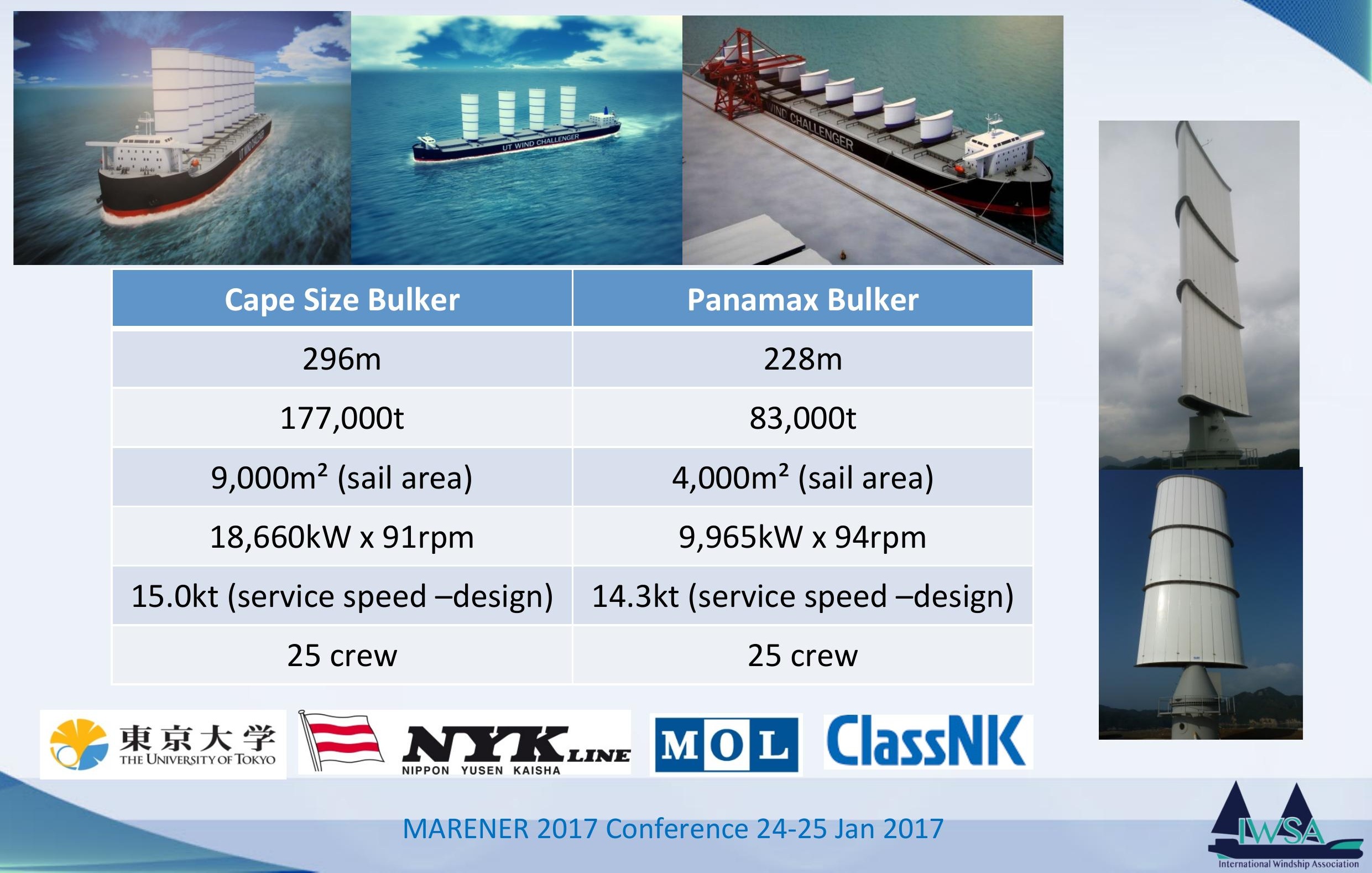




\section{Case Study: Smart Green Shipping Alliance}

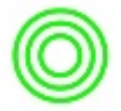

smart green shipping alliance

\section{smart}

Proven - Masts rotate to capture maximum wind. Push-button operation from the bridge ensures safety. Same crew number as conventional ship. No ropes or rigging on deck to obstruct cargo handling.

TRADEWIND automated routing system optimises wind propulsion.

Hull made from recycled steel

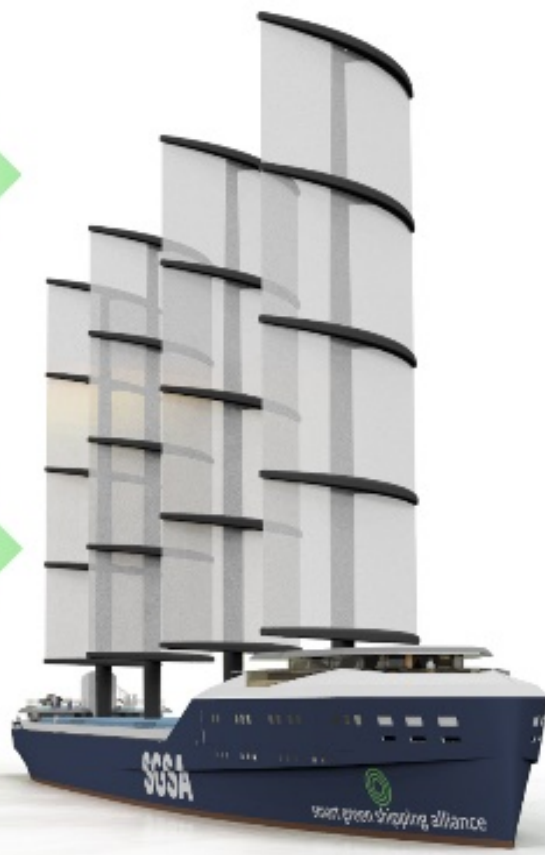

SAILING HYBRID SHIPS Potentially $100 \%$ renewable today

Commercially, technically and environmentally superior

\section{green}

At least $50 \%$ propulsion from wind

Biofuelled conventional engines ensure scheduling reliability. Biofuel bought on long term fuel supply contracts provide fuel cost certainty.
TATA STEEL

HUMPHREYS

YACHT DESIGN

WOLFSON UNIT FOR MAAINE TECHNOLOLY

10

CAMMELL LAIRD

Rolls-Royce

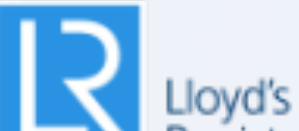

Register

Creates new transformational business model, increases order book to reduce unit cost and further improve customer proposition. 


\section{Regulation}

Clear, stable framework for low carbon shipping development worldwide.

Modern shipping practices.

\section{Finance}

Efficiency gains shared by all players. Finance decisions based on long-term infrastructure investment horizons

\section{Sustainable Shipping}

Commercial Profitability/Viability

Logistics chain - factory/farm gate to dinner plate. Circular economy - cradle to cradle development.

\section{Technical}

Vision of Low Carbon Technology. Systematic development of R\&D, demonstration \& market introduction of low carbon technologies 


\section{Any Questions?}

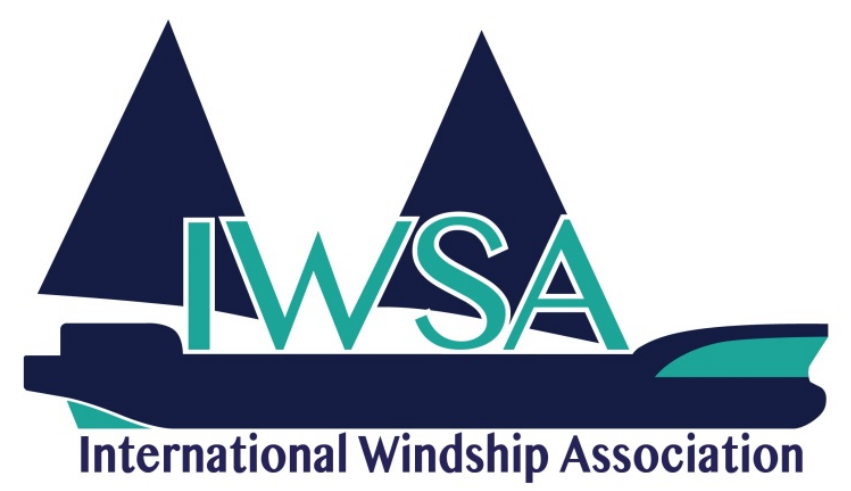

\section{International Windship}

Association www.wind-ship.org

Gavin Allwright secretary@wind-ship.org 